\title{
Origin of Current Blockades in Nanopore Translocation Experiments
}

\author{
Stefan Kesselheim, ${ }^{*}$ Wojciech Müller, and Christian Holm ${ }^{\dagger}$ \\ Institut für Computerphysik, Universität Stuttgart, Allmandring 3, D-70569 Stuttgart, Germany
}

(Received 7 August 2013; published 6 January 2014)

\begin{abstract}
We present a detailed investigation of the ionic current in a cylindrical model nanopore in the absence and the presence of a double stranded DNA homopolymer. Our atomistic simulations are capable of reproducing almost exactly the experimental data obtained by Smeets et al., including notably the crossover salt concentration that yields equal current measurements in both situations. We can rule out that the observed current blockade is due to the steric exclusion of charge carriers from the DNA, since for all investigated salt concentrations the charge carrier density is higher when the DNA is present. Calculations using a mean-field electrokinetic model proposed by van Dorp et al. fail quantitatively in predicting this effect. We can relate the shortcomings of the mean-field model to a surface related molecular drag that the ions feel in the presence of the DNA. This drag is independent of the salt concentration and originates from electrostatic, hydrodynamic, and excluded volume interactions.
\end{abstract}

DOI: 10.1103/PhysRevLett.112.018101

PACS numbers: 87.10.Tf, 87.15.ap

Nanopores are holes in membranes that can range from one to a few hundred nanometers. Observing macromolecular transport through nanopores by current measurements reveals interesting and widely applicable insights into the properties of single molecules. Especially DNA transport has been investigated as seminal results $[1,2]$ indicated a high potential for DNA sequencing. Pores can be realized by inserting pore proteins into a lipid bilayer, or by creating holes in thin synthetic membranes, so-called solid-state nanopores. Good reviews are given in Refs. [3-5].

Figure 1 shows a typical setup for a nanopore experiment. A thin membrane with a small pore separates two electrolyte filled compartments. A voltage is applied between them and the electric current is monitored. Macromolecules, in this Letter we consider doublestranded DNA, induce short modulations in the electric current when traversing the pore. These events are the main observable in the experiments.

Under most experimental conditions a decrease of the current during translocation events is observed. This seems reasonable since the presence of the DNA reduces the pore cross section. However, DNA is a highly charged macromolecule, surrounded by a cloud of neutralizing counterions that can enhance the charge transport. Both effects compete, and experimentally it was found that the electrolyte concentration governs the sign of the total effect: at high salt concentration a current reduction is observed, whereas at low salt concentrations an enhancement has been found [6-8]. Furthermore, the mobility of ions in the vicinity of a DNA molecule may be altered. Zhang

Published by the American Physical Society under the terms of the Creative Commons Attribution 3.0 License. Further distribution of this work must maintain attribution to the author(s) and the published article's title, journal citation, and DOI. and Shklovskii, e.g., introduced a friction coefficient between ions and DNA [9], and in Ref. [10] a reduction of the diffusion coefficient near DNA bases was measured in atomistic simulations.

Until now the interplay of these effects has not been fully understood. In this Letter we investigate the problem with the help of atomistic molecular dynamics simulations. The conductivity of a nanopore at different electrolyte concentrations with and without an inserted DNA is measured. Our results show quantitative agreement with the experiments of Smeets et al. [6], and moreover, a detailed comparison of our results to an electrokinetic continuum model reveals that the ions in the vicinity of the DNA are slowed down considerably when compared to the bulk. Our findings show that the blockades observed in translocation experiments are largely caused by frictional forces

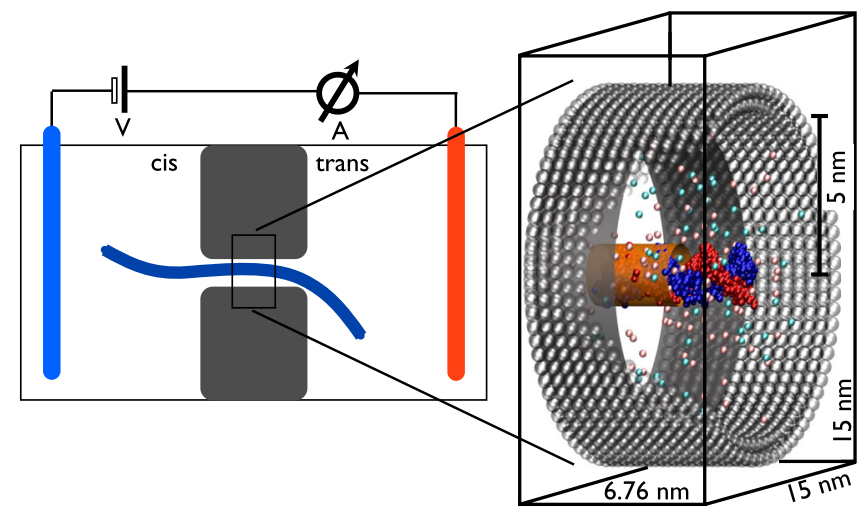

FIG. 1 (color online). Left panel: Sketch of a typical DNA translocation experiment. DNA (blue contour) is driven through the pore by applying a voltage. Right panel: We model only the central region of an long cylindrical pore. It contains a DNA homopolymer centered on the axis, a variable $\mathrm{KCl}$ concentration, and is filled with explicit water molecules. 
between the DNA and salt ions, and not by a reduction of the pore cross section.

In the conductance measurements of Ref. [6] the influence of the electrolyte concentration was investigated systematically for $10 \mathrm{~nm}$ wide nanopores in a silicon wafer. Double-stranded $\lambda$-DNA was transported through a nanopore and the electric current was measured. The same experiment was performed for different electrolyte concentrations between 0.1 and $1 \mathrm{~mol} / 1$ potassium chloride. For the lowest salt concentrations a current increase of $15 \%$ was observed, while for a unimolar electrolyte conditions a blockade of $\sim 4 \%$ was found. The crossover point between enhancement and blockade was determined to be around $0.3 \mathrm{~mol} / 1$.

In a similar experimental setup it was possible to determine the force that is electrically exerted on the DNA. Several $(\lambda)$-DNA strands were attached to a colloidal particle that could be positioned by laser tweezers close to the nanopore opening. Single DNA strands could reversibly be inserted into the pore by applying a voltage of $100 \mathrm{mV}$. The force applied on the particle by the DNA was inferred from the displacement of the trapped colloid [11]. The observed forces were explained with the following continuum model proposed by van Dorp et al. [12]: the DNA was treated as an infinitely long homogeneously charged cylinder with a radius of $1.1 \mathrm{~nm}$ and a line charge density $\lambda$ of $5.88 e / \mathrm{nm}$. This corresponds to two elementary charges per base pair every $0.34 \mathrm{~nm}$ along the DNA axis. The motion of the surrounding ions and water molecules was described by the standard model of electrokinetics [13,14]: the Poisson equation for electrostatics, the Stokes equation for fluid flow and diffusion-convection equations for two ion species, $\mathrm{K}^{+}$and $\mathrm{Cl}^{-}$. In the simple cylinder-in-cylinder geometry the local ion concentrations $c^{ \pm}(r)$ can be obtained directly from a solution of the Poisson-Boltzmann equation, as the distribution of ions is not perturbed by a field applied along the cylinder axis. The Stokes equation can be solved by simple integration to obtain the axial water velocity $u$. Finally, the ion velocities $v^{ \pm}$are determined from their respective mobility $\mu^{ \pm}$times the electric field plus the fluid velocity, $v^{ \pm}=\mu^{ \pm} E+u$.

We solved this model numerically and found that it fails to describe the observed conductance crossover (see Supplemental Material [15]). Only for electrolyte concentrations above $0.8 \mathrm{~mol} / 1$ the number of ions excluded from the pore due to the volume of the DNA exceeds the number of extra ions in the counterion cloud. Taking into account the motion of water even shifts the crossover to $1.2 \mathrm{~mol} / \mathrm{l}$. This discrepancy motivated us to perform simulations with atomistic resolution. Our strategy is to create a system that can be compared easily to the continuum model, so that we can assess its validity.

Our setup is depicted in Fig. 1. A pore of length $6.76 \mathrm{~nm}$ and radius $5 \mathrm{~nm}$ was created by placing two layers of Lennard-Jones particles with $\sigma=0.5 \mathrm{~nm}$ at mutual distances of $0.5 \mathrm{~nm}$ on a cylinder in a simulation box of $15 \times 15 \times 6.76 \mathrm{~nm}^{3}$. Periodic boundary conditions are applied in the axial direction, so that the pore resembles a section of a long cylindrical pore. We added a DNA consisting of $20 \mathrm{CG}$ base pairs, corresponding to two full turns of the helix. Bonds were not only created between adjacent bases, but also between the first and the last base pair so that the resulting strand has no end. Water molecules and ions were added to the system and it was checked that the pore wall did not allow any water molecules or ions to leak out. The DNA charge was neutralized by $40 \mathrm{~K}^{+}$ions and a varying number between 16 and 320 of $\mathrm{KCl}$ pairs were added to the system as salt ions. The phosphorous atoms of the DNA as well as the pore beads were fixed in space with harmonic springs, reflecting the experimental observation that the DNA motion is much slower than the ion motion. Water was added to the pore and an electric field of $0.2 \mathrm{~V} / \mathrm{nm}$ was applied in axial direction. The ions start moving along the pore and particles leaving the system on one side enter on the other side. With this setup we neglect DNA conformational changes, that are important for thinner pores of finite length [16]. On the other hand we reproduce the continuum model geometry precisely, only with atomistic resolution, and hence can easily assess its accuracy and deficits.

We applied the AMBER03 force field [17] and the SPC/E water model [18]. For the potassium ions we applied the force field parameters suggested by Dang [19-21]. This parameter set was proven to reproduce the structure of a DNA dodecamer well [22]. The bonds connecting hydrogens atoms to the DNA were held fixed using the P-LINCS algorithm [23]. A stochastic velocity rescaling thermostat [24] kept the temperature of the system constant. The coupling time was set to 5 ps. Electrostatic interactions were calculated using the SPME method [25] with a grid constant of $0.125 \mathrm{~nm}$. Ten independent runs of five nanoseconds were performed for every salt concentration. The first nanosecond of every run was discarded to ensure that the system had reached a stationary state independent of the initial configuration. We used the software GROMACS 4.5.5 [26-28]. Conceptually similar simulation studies have been conducted by Luan et al. [29], however, with a focus on measuring the force acting on the DNA.

In the following we perform a detailed investigation of the static and dynamic properties of the system. It is split into two parts: First the density profiles $c^{ \pm}(r)$ and velocity profiles $v^{ \pm}(r), u(r)$ are compared to the continuum model. Second we determine the electric current through the pore and compare this to the experimental results of [6].

The static ion density profile reflects the typical behavior from the literature (e.g., [29-31]). Figure 2 shows, as an example, the simulation with 64 added ion pairs added. The counterion density exhibits two significant peaks. One peak occurs at $r=0.5 \mathrm{~nm}$ (full height not shown) and a second peak is observed at $r=1.25 \mathrm{~nm}$. The first peak is 

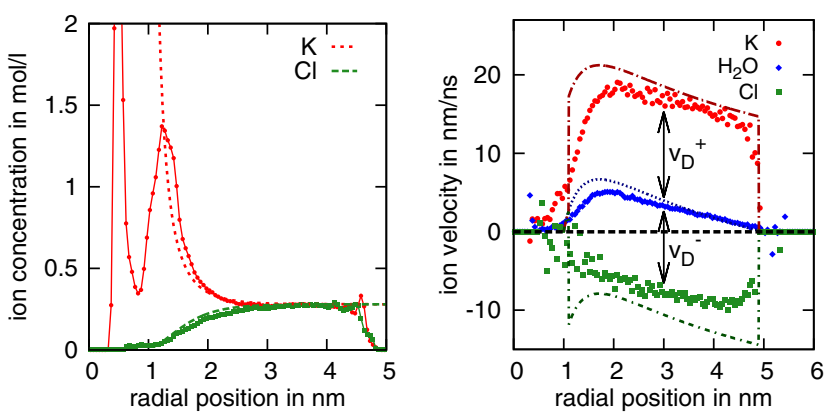

FIG. 2 (color online). Left panel: Comparison of the ion concentration as a function of the distance $r$ from the pore axis at around $0.3 \mathrm{~mol} / 1$ of the atomistic data (points) to the continuum model (broken lines). The long distance decay of the second peak at $r=1.25 \mathrm{~nm}$ is well described by the continuum model. Right panel: Comparison of the measured ion and water velocities as a function of $r$.

related to counterions entering into the major and minor grove of the DNA. This peak compensates $1 / 3$ of the DNA's bare charge at all considered salt concentrations. The second peak indicates the effective radius of the DNA backbone. For larger distances than $1.25 \mathrm{~nm}$, the ion distribution is well described by the continuum model. In the region between $r=3 \mathrm{~nm}$ and $r=4 \mathrm{~nm}$ an almost complete screening of the DNA charge was observed for all salt concentrations. We did not explicitly consider ion reservoirs in our simulations, but the concentration of a hypothetically attached reservoir can be obtained from the mean salt concentration in this region (see the Supplemental Material [15] for details). We hence determined the effective reservoir concentration a posteriori for a given number of ion pairs added to the system.

In the right panel of Fig. 2 we display the ion velocities $v^{ \pm}$of both ion species and the water velocity $u$ as a function of the radial position $r$. The electrokinetic model (dotted curves) and the atomistic simulation data are qualitatively similar. An electro-osmotic flow of water is observed in the migration direction of the $\mathrm{K}^{+}$ions. Both ion species move significantly faster than the water whose velocity tends to zero in the atomistic model approximately at the inner boundary of the continuum model. The agreement of the hydrodynamic no-slip boundary between both models up to the size of a water molecule is remarkable.

Motivated by the simple decomposition of the ion velocity $v^{ \pm}=\mu^{ \pm} E+u$ in the continuum model, we define a position dependent mobility of the ions by computing their actual velocity minus the local water velocity, $\mu(r)^{ \pm}=\left(v^{ \pm}-u\right) / E$. In the region farthest away from both the pore wall and the DNA the mobility is constant, and we denote this constant value by $\mu_{D}$, the free mobility. Note, however, that this value is concentration dependent, reflecting the known fact that the specific conductance is concentration dependent, as explained quantitatively by Onsager [32]. In the left panel of Fig. 3 we show the
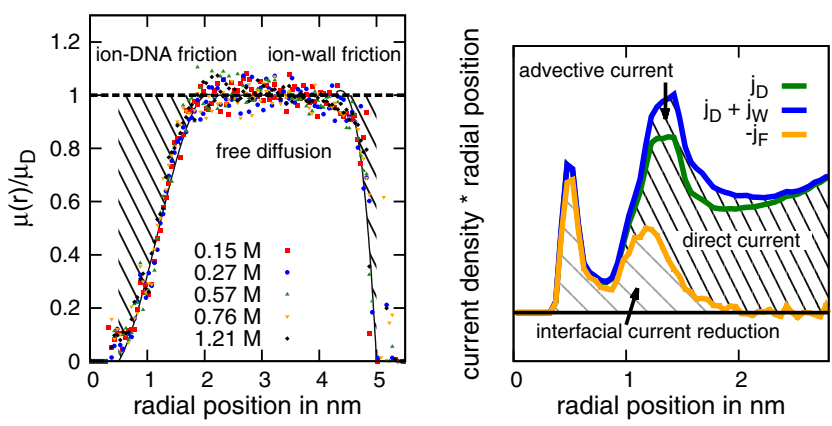

FIG. 3 (color online). Left panel: position dependent ion mobility normalized by the bulk mobility $(v-u) / \mu_{D} E$ for five different salt concentration. Near the DNA and the wall a significant reduction is observed. Right panel: Observed current densities as a function of $r$. We distinguish the direct current $j_{D}$, the advective current $j_{W}$, and the (negative) frictional current $j_{F}$. The direct current near the DNA is largely canceled by friction.

position dependent $\mathrm{K}^{+}$mobility for electrolyte concentrations between 0.15 and $1.2 \mathrm{M}$, normalized by $\mu_{D}$. All curves collapse onto a single master curve. The range of the mobility reduction is comparable with the effect found in Ref. [10]. The different geometries, however, make a direct comparison difficult.

We interpret the mobility reduction at the boundary as being caused by three factors. First, the major and minor groove produce a microscopically rough DNA surface, so that ions are trapped inside are virtually immobile. Second, the charge pattern on the DNA causes electrofriction $[33,34]$ that can extend beyond the DNA backbone. Finally, it is well known that hydrodynamic interactions slow down the motion of particles near a hydrodynamic boundary [35], an effect that is likely important on the nanoscale as well.

In order to quantify how the mobility reduction next to the boundary affects the current, we split the current into three contributions: (1) the current $I_{D}$ that would be expected if the mobility was constant and the water was immobile, (2) the advective current $I_{W}$ due to the water flow, and (3) the (negative) current contribution $I_{F}$ that is suppressed by interfacial friction. We express all of these in terms of current densities which we define as the direct current density $j_{D}=c^{+} \mu_{D}^{+} E-c^{-} \mu_{D}^{-} E$, the advective current density (as in [36]) $j_{W}=c^{+} u-c^{-} u$, and the interfacial current reduction density $j_{F}=j-j_{D}-j_{W}$, where $j$ is the total current density. The direct current depends, apart from the concentration dependence of the mobility, only on the number of ions of both species inside the pore. Therefore it contains both the extra counterions that the DNA brings into the pore, and the ions expelled from the pore due to the DNA's finite volume.

In the right panel of Fig. 3 we report the direct and advective current density, the density of interfacial current reduction. They are weighted by the radius, so that the area under the graph corresponds to the magnitude of the 


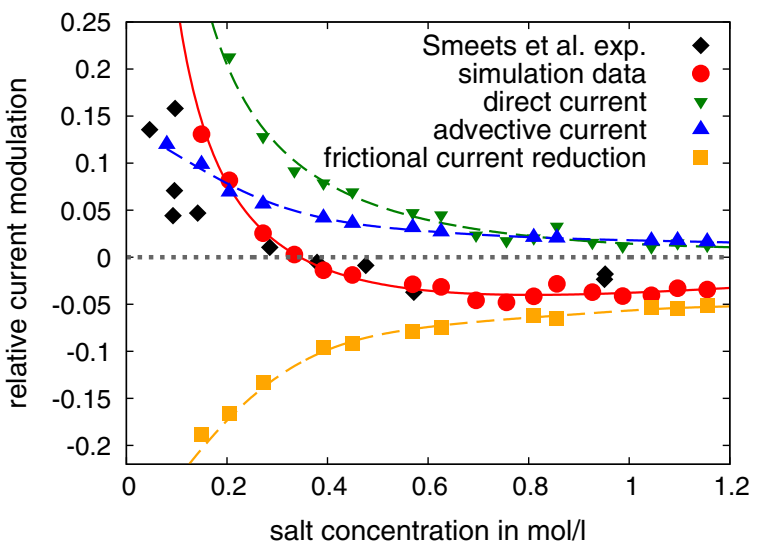

FIG. 4 (color online). Relative current modulation caused by the DNA as a function of salt concentration for the experiments (diamonds) and simulations (circles). The quantitative agreement between simulations and experiments is good, especially the location of the crossover point between enhancement and reduction. We decomposed the total current into direct current (downward triangles), advective current (upward triangles), and friction (squares) and consider their contribution to the modulation independently. The current reduction due to friction is responsible for observing an overall current reduction at large salt concentrations.

associated current. The area between the $j_{F}$ and the sum of $j_{D}$ and $j_{W}$ represents the total current. The shape of the direct current follows exactly the ion concentration reported in Fig. 2. The advective current $j_{W}=$ $\left(c_{+}-c_{-}\right) u$ is nonzero only in the regions with net charge, namely the Debye layer. It only occurs if a DNA is in the pore, and is a positive modulation of the total current. In proximity of the DNA the conduction is largely suppressed. The fact that ions in the DNA's grooves are virtually immobile is reflected in $j_{D}$ and $j_{F}$ being almost equal. This, however, applies only to $1 / 3$ of the counterions. Also ions further away from the pore axis, at $r>$ $1 \mathrm{~nm}$, are considerably reduced in mobility, resulting in an larger frictional current reduction.

We performed identical simulations also for a DNA-free pore. We investigate the relative current modulation $\left(I_{\text {with DNA }}-I_{\text {without DNA }}\right) / I_{\text {without DNA }}$ as this quantity depends neither on the length of the investigated pore nor on the absolute value of the mobility of the ions. The total current was calculated from the displacement of the ions during a simulation run. In Fig. 4 the results are displayed together with the experimental data where the lines are only guides to the eye. We decompose our data into a direct, an advective, and a frictional component. For all salt concentrations the current modulations from the simulation are in good agreement with the experimental data. For high salt conditions a current reduction of around 5\% is observed. The experiments suggest a somewhat lower reduction, however with uncertainties similar to the deviations between experiments and simulation. For low salt concentrations the predicted enhancement is larger than experimentally observed. This can be justified by noting that the nanopores are not very long (around $40 \mathrm{~nm}$ ) and have an hourglass shape. Only the radius at the constriction corresponds to the $10 \mathrm{~nm}$ used here. When the pore is wider the relative enhancement or blockade can be expected to be smaller. The crossover point between enhancement and blockade occurs at an electrolyte concentration of $0.3 \mathrm{~mol} / \mathrm{l}$, agreeing with the experiments.

The observed direct current is always larger in the presence of the DNA since the number of ions in the pore at equal reservoir concentration is always larger with DNA in the investigated concentration range up to $1.2 \mathrm{~mol} / \mathrm{l}$. The continuum model predicts this only to be the case up to $0.8 \mathrm{~mol} / \mathrm{l}$. Thus the obstruction of the cross section due to the DNA's finite volume is always overcompensated by the presence of the Debye layer. The advective contribution to the current only appears in the presence of a DNA strand and is always positive. The effect that is responsible for the crossover effect, however, can be clearly identified: without the mobility reduction at the surface of the DNA no current blockades would be observed.

To conclude, our atomistic simulation data are in excellent quantitative agreement with the experimentally observed current modulations. DNA in the pore increases the conductance for concentrations lower than around $0.3 \mathrm{~mol} / \mathrm{l}$, whereas for higher concentrations it decreases it. The current reduction is not caused by a reduction of the pore cross section. On the contrary, the number of ions in the pore is always enhanced by the DNA at the considered electrolyte concentrations. The main physical effect is the change in the ion mobility which is reduced due to a molecular friction effect near the DNA surface and in the DNA grooves. The simple electrokinetic model of [12], essentially consisting of a charged cylinder in cylindrical pore, describes the system surprisingly well. The long distance ion distribution, the ion velocity, and the magnitude of the occurring electro-osmotic flow are captured almost quantitatively correct. This encourages applying models with this degree of detail (e.g., [36]). Since the surface friction is conceptually not contained in the model, they fail in the quantitative prediction of the current reduction observed in experiments.

The authors acknowledge funding by the German Science Foundation (DFG) through the collaborative research centre SFB 716. C. H. thanks the Isaac Newton Institute for Mathematical Sciences, Cambridge, U.K., for financial support during his stay at the programme "Mathematical Modelling and Analysis of Complex Fluids and Active Media in Evolving Domains in 2013." Computing time was obtained through the Höchstleistungsrechenzentrum Stuttgart (HLRS) and the BW-Grid. We furthermore thank U.F. Keyser and his group in Cambridge for enlightening discussions, and O. A. Hickey for a critical reading of the manuscript. 
*stefan.kesselheim@icp.uni-stuttgart.de

†holm@icp.uni-stuttgart.de

[1] M. Akeson, D. Branton, J. J. Kasianowicz, E. Brandin, and D. W. Deamer, Biophys. J. 77, 3227 (1999).

[2] A. Meller, L. Nivon, E. Brandin, J. Golovchenko, and D. Branton, Proc. Natl. Acad. Sci. U.S.A. 97, 1079 (2000).

[3] C. Dekker, Nat. Nanotechnol. 2, 209 (2007).

[4] S. Howorka and Z. Siwy, Chem. Soc. Rev. 38, 2360 (2009).

[5] B. Venkatesan and R. Bashir, Nat. Nanotechnol. 6, 615 (2011).

[6] R. M. M. Smeets, U.F. Keyser, D. Krapf, M.-Y. Wu, N.H. Dekker, and C. Dekker, Nano Lett. 6, 89 (2006).

[7] H. Chang, F. Kosari, G. Andreadakis, M. A. Alam, G. Vasmatzis, and R. Bashir, Nano Lett. 4, 1551 (2004).

[8] R. Fan, R. Kamik, M. Yue, D. Li, A. Majumdar, and P. Yang, Nano Lett. 5, 1633 (2005).

[9] J. Zhang and B. I. Shklovskii, Phys. Rev. E 75, 021906 (2007).

[10] J. Comer and A. Aksimentiev, J. Phys. Chem. C 116, 3376 (2012).

[11] U. Keyser, J. van der Does, C. Dekker, and N. Dekker, Rev. Sci. Instrum. 77, 105105 (2006).

[12] S. van Dorp, U. F. Keyser, N. H. Dekker, C. Dekker, and S. G. Lemay, Nat. Phys. 5, 347 (2009).

[13] R. J. Hunter, Foundations of Colloid Science (Oxford University Press, Oxford, 2001), 2nd ed.

[14] D. F. Evans and H. Wennerström, The Colloidal Domain (Wiley-VCH, New York, 1999).

[15] See Supplemental Material at http://link.aps.org/ supplemental/10.1103/PhysRevLett.112.018101 for a discussion of the electrokinetic continuum model and the method we applied to determine the reservoir salt concentration a posteriori.

[16] A. Aksimentiev, Nanoscale 2, 468 (2010).
[17] Y. Duan, C. Wu, S. Chowdhury, M. C. Lee, G. Xiong, W. Zhang, R. Yang, P. Cieplak, R. Luo, T. Lee et al., J. Comput. Chem. 24, 1999 (2003).

[18] H. J. C. Berendsen, J. R. Grigera, and T.P. Straatsma, J. Phys. Chem. 91, 6269 (1987).

[19] D. E. Smith and L. X. Dang, J. Chem. Phys. 100, 3757 (1994).

[20] L. X. Dang and P. A. Kollman, J. Phys. Chem. 99, 55 (1995).

[21] L. X. Dang, J. Am. Chem. Soc. 117, 6954 (1995).

[22] A. Noy, I. Soteras, F. J. Luque, and M. Orozco, Phys. Chem. Chem. Phys. 11, 10596 (2009).

[23] B. Hess, J. Chem. Theory Comput. 4, 116 (2008).

[24] G. Bussi, D. Donadio, and M. Parrinello, J. Chem. Phys. 126, 014101 (2007).

[25] U. Essmann, L. Perera, M. L. Berkowitz, T. Darden, H. Lee, and L. Pedersen, J. Chem. Phys. 103, 8577 (1995).

[26] B. Hess, C. Kutzner, D. van der Spoel, and E. Lindahl, J. Chem. Theory Comput. 4, 435 (2008).

[27] D. V.D. Spoel, E. Lindahl, B. Hess, G. Groenhof, A. E. Mark, and H. J. C. Berendsen, J. Comput. Chem. 26, 1701 (2005).

[28] H. J. C. Berendsen, D. van der Spoel, and R. van Drunen, Comput. Phys. Commun. 91, 43 (1995).

[29] B. Luan and A. Aksimentiev, Phys. Rev. E 78, 021912 (2008).

[30] M. Feig and B. M. Pettitt, Biophys. J. 77, 1769 (1999).

[31] C. Maffeo, R. Schöpflin, H. Brutzer, R. Stehr, A. Aksimentiev, G. Wedemann, and R. Seidel, Phys. Rev. Lett. 105, 158101 (2010).

[32] L. Onsager, Chem. Rev. 13, 73 (1933).

[33] R. R. Netz, Phys. Rev. Lett. 91, 138101 (2003).

[34] Y.W. Kim and R. R. Netz, J. Chem. Phys. 124, 114709 (2006).

[35] J. R. Blake, Math. Proc. Cambridge Philos. Soc. 70, 303 (1971).

[36] P. Rowghanian and A. Y. Grosberg, J. Chem. Phys. 139, 024902 (2013). 